\title{
Menarca e antropometria em atletas adolescentes de pentatlo moderno
}

http://dx.doi.org/10.11606/1807-5509201900030433

\section{Resumo}

A menarca é um importante indicador da maturação sexual e sua ocorrência pode variar de acordo com as características antropométricas e do treinamento de modalidades esportivas. Os objetivos foram: comparar a idade da menarca e a antropometria em grupos de atletas adolescentes de pentatlo moderno que iniciaram o treinamento antes e após a menarca; analisar as relações entre o tempo pósmenarca com a antropometria em grupos de atletas adolescentes de pentatlo moderno que iniciaram o treinamento antes e após a menarca; analisar as relações entre a idade cronológica e a antropometria em atletas adolescentes de pentatlo moderno não menarqueadas. Participaram 31 atletas menarqueadas $(14,69 \pm 2,03 a n o s$ de idade) e 15 não menarqueadas $(12,22 \pm 1,07$ anos de idade). Foram avaliadas a idade da menarca, tempo pós-menarca, medidas antropométricas e caracteristicas do treinamento. Utilizou-se a estatística descritiva, comparações para amostras independentes e a correlação de Pearson $(p<0,05)$. A idade da menarca foi $11,80 \pm 0,95$ anos. Após divisão do grupo das menarqueadas, diferença significativa $(p=0,006)$ foi observada na idade da menarca entre meninas que iniciaram o treinamento antes $(12,62 \pm 0,95)$ e após $(11,42 \pm 1,17)$ a menarca. A análise descritiva permitiu observar a idade cronológica do grupo não menarqueadas sendo maior que a idade da menarca no grupo das menarqueadas, assim como foi maior o tempo de treinamento e menor a adiposidade. A idade de início do treinamento aparenta ser o maior responsável pelas características da menarca apresentadas em pentatletas, sem descartar possíveis efeitos do baixo percentual de gordura.

Palavras-chave: Maturidade Sexual; Antropometria; Ciclo Menstrual; Esportes.

\section{Introdução}

O primeiro período menstrual da mulher chamase menarca, e no desporto constitui uma importante informação para avaliar o estado maturacional ou a saúde de atletas adolescentes em diferentes modalidades esportivas ${ }^{1}$. Em geral, a idade de ocorrência da menarca (IM) pode estar associada à genética, individualidade biológica, às condiçôes ambientais, ao estado socioeconômico e nutricional de uma população ${ }^{2-4}$.

O treinamento físico é um dos fatores que está associado às alteraçóes na IM. Atletas adolescentes de determinadas modalidades esportivas tendem apresentar baixo percentual de gordura corporal, que pode levar a alteraçóes hipotalâmicas, influenciando na liberação dos hormônios sexuais femininos e, consequentemente, na ocorrência atrasada da menarca ${ }^{4}$. Estudos adicionais investigaram a ação da leptina, um hormônio produzido principalmente pelo tecido adiposo e em menor proporção pelo estômago, placenta e mamas, que atua como estimulador do eixo hipotalâmico e influência na IM. Em atletas, o balanço energético negativo decorrente do exercício intenso associado à má nutrição, tem relação com baixas concentraçôes de leptina plasmática e desregulagem do sistema reprodutório $^{5-7}$.

No entanto, estudos demonstram influência do treinamento intenso em idade precoce no 
atraso da menarca, independente da quantidade de gordura corporal ${ }^{8,9}$. Vale ressaltar, ainda, que outras características antropométricas, como medidas que predispóem a seleção natural para o desporto, destacando a maior estatura e a medida de quadril reduzida, e o estresse inerente à prática desportiva ${ }^{1,7,10}$, também podem influenciar no atraso da menarca de atletas.

Desta forma, comumente são investigados os efeitos causados pelo treinamento intenso e pela antropometria na IM em diferentes modalidades esportivas $^{11-13}$. Ademais, por possibilitar avaliar a precocidade ou o atraso da maturação sexual, a menarca pode auxiliar no planejamento do treino de jovens atletas, pois meninas de mesma idade cronológica podem estar em momentos maturacionais diferentes, influenciando no desempenho motor e na antropometria, devido às alteraçóes da estatura e da composição corporal ${ }^{14-16}$. Em adendo, a idade ginecológica (IG) que é obtida pela subtração da idade cronológica pela idade da menarca, permite observaçóes das relaçóes entre o avanço maturacional e o desenvolvimento físico no período pós-menarca de atletas e não atletas ${ }^{14,17}$.

O pentatlo moderno é um desporto olímpico de origem militar, que envolve a esgrima, natação, hipismo e o combinado de corrida e tiro, exigindo uma alta carga de treinos já na fase de iniciação esportiva. Entretanto, a influência do treinamento para a prática desta modalidade esportiva na IM, assim como as relaçóes entre o estado maturacional com a antropometria, ainda não foram investigadas em adolescentes.

Nesse sentido, os objetivos deste estudo foram: 1) comparar a idade da menarca e a antropometria em grupos de atletas adolescentes de pentatlo moderno que iniciaram o treinamento antes e após a menarca; 2) analisar as relações entre a idade ginecológica e a antropometria em grupos de atletas adolescentes de pentatlo moderno que iniciaram o treinamento antes e após a menarca; e 3) analisar as relações entre a idade cronológica e a antropometria em atletas adolescentes de pentatlo moderno não menarqueadas.

\section{Método}

\section{Amostra}

A coleta de dados, autorizada pela Confederação de Pentatlo Moderno do Rio de Janeiro, foi realizada nas atletas das categorias Jovem A, B, C e $\mathrm{D}$, que correspondem às idades de 11 a 18,99 anos, nas cidades sedes dos campeonatos brasileiros de 2012 e 2013, Resende/RJ e Rio de Janeiro/ RJ, respectivamente. A FIGURA 1 apresenta o desenho da seleçáo dos sujeitos deste estudo transversal. Vale ressaltar que em casos de meninas menarqueadas que participaram das duas coletas, houve preferência para as avaliaçóes de 2012, por ser mais fácil recordar os dados da menarca com menos tempo de ocorrência. No caso das participantes não menarqueadas entrevistadas nos dois anos, houve preferência pelos dados coletados em 2013 por estarem mais próximos da menarca.

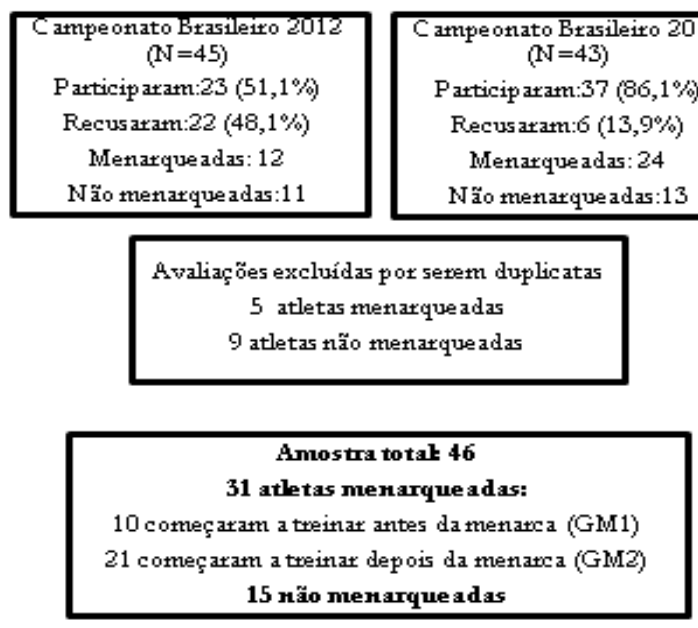

FIGURA 1- Seleção e distribuição de atletas de pentatlo moderno participantes do estudo. 


\section{Procedimentos metodológicos}

Todos os treinadores foram comunicados previamente do estudo e informaram os responsáveis e atletas sobre o seu desenvolvimento antes das competiçóes. A coleta de dados foi realizada entre o período de chegada ao hotel e o início do evento, em horários marcados de acordo com a disponibilidade pessoal da atleta.

A idade de ocorrência da menarca (IM) foi obtida pelo método "status quo", que questiona a ocorrência ou náo da menarca ${ }^{4}$. Em caso de resposta positiva foi identificado o mês e o ano de ocorrência por um questionário retrospectivo. A idade ginecológica (IG) foi obtida por meio da subtração da idade atual pela IM. Também foi reportada, em entrevista com o pesquisador, a idade de início de treinamento, quantidade de dias semanais e horas diárias de treinamento, sendo calculados o tempo de treinamento em meses e a quantidade de horas semanais treinadas.

A medição de massa corporal foi realizada em uma balança digital da marca Plenna Brasil, com precisão de $0,1 \mathrm{~kg}$. A estatura foi medida em um estadiômetro portátil da marca WCS, com precisão de $0,1 \mathrm{~cm}$. As dobras cutâneas de bíceps, tríceps, subescapular, abdominal, suprailíaca, coxa e perna medial foram tomadas por um único e experiente avaliador, sendo medidas três vezes ao lado direito do corpo com um adipômetro científico da marca Cescorf, com precisão de $0,1 \mathrm{~mm}$. Nos casos em que as medidas na mesma dobra cutânea extrapolaram o limite de 5\% de diferença das demais, uma nova série de três medidas foi realizada. $\mathrm{O}$ resultado das medidas de cada dobra cutânea foi a média aritmética das três medidas. Medidas de perímetros do quadril e cintura foram realizadas com uma trena metálica da marca Sanny, com precisão de $0,1 \mathrm{~cm}$. Os protocolos de medidas descritos por LOHMAN, Roche E MartorelL ${ }^{18}$ foram adotados em toda a avaliação antropométrica.

O percentual de gordura foi calculado por meio da equação proposta por SlaUghter et al. ${ }^{19}$, para crianças e adolescentes, utilizando as medidas de dobras cutâneas de tríceps e subescapular. Nos casos de meninas com mais de 18 anos de idade, utilizouse a equação de Durnin E Womersley $(1974)^{20}$, utilizando as dobras cutâneas de tríceps, bíceps, subescapular e suprailíaca.

O projeto foi aprovado pelo Comitê de Ética em Pesquisa do Hospital Universitário Clementino Fraga Filho com protocolo 090/2011. Os participantes que aceitaram fazer parte do estudo tiveram o Termo de Consentimento Livre e Esclarecido assinado pelo responsável.

\section{Análise estatística}

A descrição dos dados antropométricos e do treinamento das atletas menarqueadas e não menarqueadas foram analisadas por meio da média aritmética, desvio padrão e valores mínimo e máximo. Os grupos GM1 e GM2, após análise da distribuição dos dados por meio de medidas de dispersão e gráficos homogramas, foram comparados com utilização do teste t de Student para amostras independentes nas seguintes variáveis: menarca, antropometria e horas semanais de treinamento. Na variável tempo de treinamento foi utilizado o teste $\mathrm{U}$ de Mann Whitney. Em todos os procedimentos foram adotados $\mathrm{p}<0,05$ para significância.

Após análise das medidas de dispersão, a correlação de Pearson foi utilizada entre a variável IG e as características antropométricas nas atletas menarqueadas. No grupo de atletas não menarqueadas (GNM), a idade cronológica foi correlacionada com a antropometria, com adoção de $\mathrm{p}<0,05$ para significância.

Os procedimentos estatísticos foram realizados no pacote estatístico SPSS versão 20.

\section{Resultados}

As características antropométricas e do treinamento das participantes dos campeonatos brasileiros de 2012 e 2013, divididas em um grupo menarqueado e outro não menarqueado, podem ser observadas na TABELA 1.

Na TABELA 2 são apresentadas comparações entre GM1 e GM2 nas variáveis idade cronológica, IM,
IG, antropometria e tempo de treinamento. A IM foi significantemente mais elevada no GM1, provocando menor IG. Na antropometria não houve diferenças significativas nas varáveis analisadas. Entretanto, o GM1 apresentou mais tempo de treinamento de pentatlo moderno e, mesmo não sendo significativo, o p valor encontrado foi próximo a 0,05 . 
Junior SJF, et al.

TABELA 1 -Idade da menarca e ginecológica, antropometria e características do treinamento de adolescentes dos campeonatos brasileiros de pentatlo moderno de 2012 e 2013.

\begin{tabular}{|c|c|c|c|c|}
\hline & \multicolumn{2}{|c|}{$\begin{array}{l}\text { Atletas menarqueadas } \\
\qquad(\mathrm{n}=31)\end{array}$} & \multicolumn{2}{|c|}{$\begin{array}{l}\text { Atletas não menarqueadas } \\
\qquad(\mathrm{n}=15)\end{array}$} \\
\hline & Média $\pm D P$ & Mín-Máx & Média $\pm D P$ & Mín-Máx \\
\hline Idade (anos) & $14,69 \pm 2,03$ & $11,41-18,40$ & $12,22 \pm 1,07$ & $11,00-14,58$ \\
\hline IM (anos) & $11,80 \pm 1,17$ & $9,75-14,40$ & $\mathrm{x}$ & $\mathrm{x}$ \\
\hline IG (anos) & $2,89 \pm 1,83$ & $0,20-6,16$ & $\mathrm{x}$ & $\mathrm{x}$ \\
\hline MC (kg) & $53,19 \pm 7,11$ & $40,20-66,60$ & $42,24 \pm 7,71$ & $32,10-59,80$ \\
\hline Estatura $(\mathrm{cm})$ & $160,31 \pm 5,81$ & $145,02-167,00$ & $151,39 \pm 7,24$ & $135,00-163,00$ \\
\hline Cintura $(\mathbf{c m})$ & $70,53 \pm 6,64$ & $63,00-85,50$ & $66,32 \pm 6,50$ & $58,20-81,80$ \\
\hline Quadril (cm) & $88,00 \pm 6,20$ & $77,50-100,00$ & $81,65 \pm 6,23$ & $73,60-94,00$ \\
\hline$\Sigma 2 \mathrm{DC}$ MMSS (mm) & $18,49 \pm 7,05$ & $9,30-40,30$ & $15,34 \pm 5,26$ & $8,10-24,70$ \\
\hline$\Sigma 3 D C$ tronco $(\mathrm{mm})$ & $43,16 \pm 15,80$ & $23,90-83,20$ & $35,67 \pm 20,31$ & $12,30-82,20$ \\
\hline$\Sigma 2 \mathrm{DC}$ MMII (mm) & $29,55 \pm 9,10$ & $17,80-58,80$ & $24,34 \pm 7,00$ & $14,40-35,10$ \\
\hline$\Sigma 7 \mathrm{DC}(\mathrm{mm})$ & $90,53 \pm 30,90$ & $36,90-171,40$ & $75,36 \pm 30,91$ & $40,60-136,70$ \\
\hline \% Gordura & $21,14 \pm 5,07$ & $13,76-31,76$ & $18,06 \pm 6,58$ & $9,71-29,23$ \\
\hline TT (meses) & $20,18 \pm 14,87$ & $0,20-60,00$ & $25,97 \pm 11,41$ & $0,50-36,00$ \\
\hline Horas/semana & $17,97 \pm 8,50$ & $5,00-35,00$ & $14,97 \pm 6,41$ & $7,50-24,00$ \\
\hline
\end{tabular}

TABELA 2 -Idade cronológica, da menarca e ginecológica, antropometria e características do treinamento de adolescentes dos campeonatos brasileiros de 2012 e 2013, menarqueadas antes e após o início do treinamento.

IM=idade da menarca; IG=Idade ginecológica; $\mathrm{MC}=$ massa corporal; $\Sigma 2 \mathrm{DC}$ MMSS=somatório de duas dobras de membros superiores; $\Sigma 3$ DC somatório de três dobras cutâneas; $\Sigma 2 \mathrm{DC}$ MMII=somatório de duas dobras cutâneas de membros inferiores; $\Sigma \mathrm{DC}=$ somatório de sete dobras cutâneas; TT=Tempo de treinamento.

\section{(nM1 (n) 10)}

\begin{tabular}{|c|c|c|c|c|c|}
\hline & \multicolumn{2}{|c|}{ GM1 $(n=10)$} & \multicolumn{2}{|c|}{ GM2 (n=21) } & \multirow[b]{2}{*}{ P valor } \\
\hline & Média $\pm D P$ & EP & Média $\pm D P$ & EP & \\
\hline Idade (anos) & $14,61 \pm 1,90$ & 1,90 & $14,73 \pm 2,14$ & 0,47 & 0,878 \\
\hline IM (anos) & $12,62 \pm 0,95$ & 0,29 & $11,42 \pm 1,09$ & 0,23 & $0,006^{*}$ \\
\hline IG (anos) & $2,01 \pm 1,28$ & 0,40 & $3,31 \pm 1,93$ & 0,41 & $0,035^{*}$ \\
\hline MC (kg) & $55,78 \pm 7,36$ & 2,32 & $51,96 \pm 6,83$ & 1,48 & 0,186 \\
\hline Estatura (cm) & $161,27 \pm 4,59$ & 1,44 & $159,83 \pm 6,36$ & 1,39 & 0,481 \\
\hline Cintura $(\mathrm{cm})$ & $73,39 \pm 8,52$ & 2,68 & $69,16 \pm 5,25$ & 1,15 & 0,099 \\
\hline Quadril (cm) & $90,54 \pm 7,55$ & 2,38 & $86,79 \pm 5,21$ & 1,13 & 0,178 \\
\hline$\Sigma 2 \mathrm{DC}$ MMSS (mm) & $19,23 \pm 6,83$ & 2,15 & $18,13 \pm 7,29$ & 1,58 & 0,684 \\
\hline$\Sigma 3 \mathrm{DC}$ tronco $(\mathrm{mm})$ & $46,91 \pm 17,56$ & 5,54 & $41,38 \pm 15,01$ & 3,28 & 0,404 \\
\hline इ2DC MMII (mm) & $31,00 \pm 12,54$ & 3,97 & $28,86 \pm 7,19$ & 1,56 & 0,625 \\
\hline
\end{tabular}




\begin{tabular}{cccccc}
\hline & \multicolumn{2}{c}{ GM1 (n=10) } & \multicolumn{2}{c}{ GM2 $(\mathbf{n = 2 1})$} & EP valor \\
\cline { 2 - 5 } & Média \pm DP & EP & Média \pm DP & EP & 0,064 \\
\hline \%gordura & $22,44 \pm 5,32$ & $1,6,96$ & $87,37 \pm 29,32$ & 6,40 & 0,625 \\
TT (meses) & $27,59 \pm 14,23$ & 4,50 & $20,53 \pm 4,98$ & 1,09 & 0,087 \\
Horas/semana & $15,84 \pm 7,29$ & 2,31 & $16,63 \pm 14,16$ & 3,09 & 0,314 \\
\hline
\end{tabular}

${ }^{*} \mathrm{p}<0,05$ para diferença significante; GM1=Grupo menarqueado antes de iniciar o treinamento; GM2=Grupo menarqueado após iniciar o treinamento; $\mathrm{IM}=$ idade da menarca; $\mathrm{IG}=$ Idade ginecológica; $\mathrm{MC}=$ massa corporal; $\Sigma 2 \mathrm{DC}$ MMSS=somatório de duas dobras de membros superiores; $\Sigma$ 3DC somatório de três dobras cutâneas; $\Sigma 2 \mathrm{DC}$ MMII=somatório de duas dobras cutâneas de membros inferiores; $\Sigma \mathrm{DC}=$ =somatório de sete dobras cutâneas cutâneas; TT = Tempo de treinamento.

Os resultados das correlações entre IG e idade cronológica com a antropometria são apresentados na TABELA 3. No GM1 o coeficiente de correlação (r) encontrado com a massa corporal (MC) foi baixo e o p valor não significativo. No entanto, o valor do coeficiente de correlação encontrado com a estatura foi alto. No GM2 o coeficiente encontrado foi alto e significativo com a MC. A correlação com a estatura apresentou forte e significativo coeficiente, embora mais baixo que no GM1.

Vale observar, ainda, que o coeficiente de correlação foi mais alto e significativo entre IG e a medida de perímetro de quadril no grupo GM1, mais baixo no grupo GM2 e negativo na correlação com a idade cronológica do grupo de atletas não menarqueadas. Nos três grupos coeficientes negativos foram observados nos somatórios de dobras cutâneas. Coeficientes negativos mais altos foram observados nas correlações com o somatório de dobras cutâneas de membros inferiores que de outras regiōes do corpo. O coeficiente de correlação entre a idade cronológica e a estatura no GNM foi mais baixo que dos grupos com menarca.

TABELA 3-Correlação entre a idade ginecológica e a antropometria em menarqueadas e entre a idade e a antropometria em não menarqueadas dos campeonatos brasileiros de 2012 e 2013.

\begin{tabular}{|c|c|c|c|c|c|c|}
\hline & \multicolumn{2}{|c|}{$\begin{array}{c}\text { GM1 }(n=10) \\
\text { IG (anos) } \\
2,01 \pm 1,28\end{array}$} & \multicolumn{2}{|c|}{$\begin{array}{c}\text { GM2 }(\mathrm{n}=21) \\
\text { IG(anos) } \\
3,31 \pm 1,93\end{array}$} & \multicolumn{2}{|c|}{$\begin{array}{c}\text { GNM (n=15) } \\
\text { IC(anos) } \\
12,22 \pm 1,07\end{array}$} \\
\hline & $\mathbf{r}$ & $\mathbf{p}$ & $\mathbf{r}$ & $\mathbf{p}$ & $\mathbf{r}$ & $\mathbf{p}$ \\
\hline MC (kg) & 0,199 & 0,581 & 0,620 & $0,003^{*}$ & 0,130 & 0,643 \\
\hline Estatura (cm) & 0,713 & $0,021^{*}$ & 0,541 & $0,026^{*}$ & 0,400 & 0,139 \\
\hline Cintura $(\mathbf{c m})$ & 0,033 & 0,924 & 0,061 & 0,793 & $-0,212$ & 0,449 \\
\hline Quadril (cm) & 0,297 & 0,376 & 0,440 & $0,046^{*}$ & $-0,072$ & 0,797 \\
\hline$\Sigma 2 \mathrm{DC}$ MMSS (mm) & $-0,192$ & 0,571 & $-0,056$ & 0,397 & $-0,298$ & 0,280 \\
\hline$\Sigma 3 \mathrm{DC}$ tronco $(\mathrm{mm})$ & $-0,078$ & 0,819 & $-0,061$ & 0,792 & $-0,145$ & 0,607 \\
\hline$\Sigma 2 \mathrm{DC}$ MMII (mm) & $-0,249$ & 0,460 & $-0,229$ & 0,317 & $-0,371$ & 0,174 \\
\hline$\Sigma 7 \mathrm{DC}(\mathrm{mm})$ & $-0,343$ & 0,332 & $-0,071$ & 0,898 & $-0,230$ & 0,410 \\
\hline \%gordura & $-0,211$ & 0,559 & $-0,068$ & 0,770 & $-0,193$ & 0,491 \\
\hline
\end{tabular}

${ }^{*} \mathrm{p}<0,05$ para significância; GM1=Grupo menarqueado antes de iniciar o treinamento; GM2=Grupo menarqueado após iniciar o treinamento; GNM=Grupo não menarqueado; IG=Idade ginecológica; IC=Idade cronológica; $\mathrm{MC}=$ massa corporal; $\Sigma 2 \mathrm{DC}$ MMSS=somatório de duas dobras de membros superiores; $\Sigma 3 \mathrm{DC}$ somatório de três dobras cutâneas; $\Sigma 2 \mathrm{DC}$ MMII=somatório de duas dobras cutâneas de membros inferiores; $\Sigma 7 \mathrm{DC}=$ somatório de sete dobras cutâneas. 


\section{Discussão}

O presente estudo inicialmente analisou diferenças descritivas de atletas menarqueadas e não menarqueadas. Menor adiposidade, que pode estar associada principalmente ao momento maturacional, foi observada no grupo não menarqueado (GNM). Outras características descritivas importantes observadas no GNM foram o tempo maior destinado ao pentatlo moderno, mesmo tendo idade cronológica mais baixa, e a idade cronológica maior que a IM verificada no grupo menarqueado.

$\mathrm{Na}$ análise do GM1 e GM2 foi observado que não há diferença de idade cronológica entre os dois grupos. No entanto, a IM foi significantemente mais elevada no GM1. Os resultados obtidos parecem estar relacionados ao início do treinamento antes da ocorrência da menarca, sendo parecidos com os encontrados por SCHTSCHERbina et al. ${ }^{8}$, que observaram em nadadoras adolescentes competitivas, que a idade do início do treinamento e não a composição corporal tem maior influência na IM.

As análises das correlações entre a IG e a idade cronológica com a antropometria demonstraram importantes características de desenvolvimento físico de atletas submetidas ao treinamento intenso de pentatlo moderno. Nos grupos GM1 e GM2 foi observado que a estatura apresenta tendência de aumento no período pós-menarca, possivelmente por ainda estarem na fase do pico de velocidade da estatura (PVE). No GNM, os resultados mais discretos podem estar relacionados ao PVE ainda não estar presente em algumas atletas ${ }^{4}$.

A tendência de aumento da massa corporal, sem o aumento da adiposidade no GM2 e com tendência de redução no GM1 e GNM, indica a redução da gordura corporal com o avanço maturacional e o aumento da massa magra. Deve-se considerar que a amostra reduzida do GM1 pode ter contribuído com um coeficiente mais baixo. No caso das meninas não menarqueadas, fica a dúvida se esta já é uma característica antropométrica relacionada à menarca tardia ou se com o avançar maturacional esta característica será alterada. Vale alertar que os menores coeficientes encontrados no GM2 podem ter sidos influenciados por pouco tempo de treinamento de alguns participantes do estudo, visto o alto desvio padrão da média aritmética no tempo de treinamento. Os resultados obtidos na medida de quadril, embora mais discretos, também parecem ter relações com a menarca tardia.
Tais observações para essas possíveis influências da antropometria e da composição corporal, aumentam ainda mais a abrangente discussão sobre este marco da puberdade em atletas adolescentes. Os resultados observados demonstram a necessidade da equipe multidisciplinar monitorar por meio de avaliações esporádicas as disfunções menstruais e, consequentemente, a saúde de atletas adolescentes ${ }^{8}$.

Em modalidades como balé clássico, ginástica rítmica ou provas de endurance, ocorre a necessidade de um baixo percentual de gordura, aumentando os riscos de desordens alimentares e até mesmo da perda da massa óssea e de disfunções menstruais, caracterizando a tríade da mulher atleta ${ }^{12,21,22}$. Desta forma, atletas adolescentes de determinadas modalidades esportivas apresentam idade da menarca tardia ${ }^{9,12}$. Mesmo na natação, desporto em que não há maiores preocupações com um percentual de gordura muito baixo, o estudo de SANTOS, LEANDRO E GUIMARÁES ${ }^{13}$ mostrou IM mais tardia em atletas de nível estadual do que em grupo controle.

No Brasil, a média da IM relatada na revisão sistemática de KLUG E FOnSECA ${ }^{1}$ foi de 12,20 anos de idade em meninas não atletas. Ao comparar com a média de 11,88 anos de idade encontrada em nosso estudo, parece que a prática do treinamento para o pentatlo moderno não influenciou na IM. Entretanto, ao levar em consideração as atletas que iniciaram o treinamento antes da menarca, a média encontrada foi de 12,62 anos de idade, ou seja, acima da relatada na população brasileira de não atletas e diferente significativamente da média de idade de 11,42 anos em meninas menarqueadas antes de iniciarem o treinamento.

As características antropométricas no que tange a adiposidade demonstram que o grupo não menarqueado pode estar sendo influenciado pela baixa quantidade de gordura corporal. Vale ressaltar que a importância de $22 \%$ de gordura relativa para a ocorrência de ciclos menstruais normalizados e $17 \%$ para a IM $^{23}$. Os valores de percentual de gordura encontrados neste estudo nas meninas não menarqueadas demonstram que parte estava abaixo do valor que resulta em alteraçôes na IM.

Claessens et al. ${ }^{9}$ também observaram na elite mundial de remadoras, maior IM em meninas que iniciaram o treinamento antes da menarca quando comparadas as que iniciaram após, não observando influências da antropometria. Entretanto, assim 
como o estudo de SCHTSCHERBina et al. ${ }^{8}$, não elucida os fenômenos que podem levar as características do treinamento e não a gordura corporal serem responsáveis por disfunçõos menstruais de atletas.

Ao levar em consideração os estudos que analisam a tendência mundial de redução da IM, dentre os possíveis influenciadores da menarca mais tardia nas atletas investigadas, podem estar os fatores psicossociais relacionados à prática desportiva, devido, principalmente, a comportamentos específicos de atletas durante a adolescência e ao estresse gerado pela necessidade de resultados no período pré-menarca ${ }^{1-3,10,24}$.

Outras possíveis explicações para estes resultados estão direcionadas a ação da leptina na função reprodutora, visto que sua produção reduzida em atletas está relacionada ao déficit de energia, redução da gordura corporal e demais características da natural seleção esportiva ${ }^{25}$. Vale ressaltar que a gordura localizada especificamente nas regiōes coxofemural produz quantidades maiores de leptina em relação à região central e, que a gordura localizada na região do quadril pode ser um importante preditor da leptina plasmática ${ }^{26}$.

Neste estudo, a distribuição da adiposidade não parece ter influenciado na IM, ao levar em consideração o somatório das dobras cutâneas em diferentes regióes do corpo e a medida de perímetro de cintura. Entretanto, o perímetro de quadril apresentou, mesmo que apenas moderado, maior coeficiente de correlação com a IG no GM2, grupo que a menarca foi mais precoce. Importante destacar a redução da gordura subcutânea de membros inferiores com o avanço maturacional e idade cronológica, parecendo ser esta uma característica de meninas submetidas ao treinamento. Novas investigações dessas medidas parecem ser importantes.

Demais características antropométricas não apresentaram resultados que merecessem destaque. Com respeito à estatura, embora haja a hipótese de meninas que estejam acima da média apresentem menarca mais tardia ${ }^{28}$.

Tanto as medidas de dobras cutâneas quanto a utilização de equações preditivas para análise do percentual de gordura não desenvolvidas ou validadas para atletas, foram limitações deste estudo, parecendo necessárias novas investigações com métodos de avaliação padrão ouro para avaliar a composição corporal e permitir uma análise mais detalhada da influência da adiposidade e até mesmo da distribuição da gordura corporal por regiōes na IM.

Em suma, a idade da menarca foi baixa ao levar em consideração que o pentatlo moderno exige treinamentos intensos. Em adolescentes que iniciaram o treinamento antes da menarca, a ocorrência foi em idade mais elevada, não havendo influência da antropometria e composição corporal. As meninas não menarqueadas apresentaram idade cronológica mais elevada do que a média geral da idade da menarca, assim como menor adiposidade que as menarqueadas, podendo haver influência da composição corporal neste grupo. Desta forma, a idade de início do treinamento aparenta ser a maior responsável pela maior idade de ocorrência da menarca em pentatletas, sem descartar possíveis efeitos do baixo percentual de gordura. São necessárias investigações futuras no âmbito das influências psicossociais do treinamento desportivo, da gordura corporal com sua distribuição e, consequentemente, da ação da leptina na ocorrência da menarca de atletas.

\title{
Agradecimentos
}

À Confederação Brasileira de Pentatlo Moderno pelo apoio em todo o processo de coleta de dados.

\begin{abstract}
Menarche and anthropometry in adolescents athletes of modern penthatlon

The menarche is an important indicator of sexual maturity and its occurrence can vary according to the anthropometric characteristics and training of sports. The objectives were: to compare the age of menarche and anthropometry in groups of modern pentathlon adolescents who started training before and
\end{abstract}


after menarche; To analyze the relationships between postmenarcheal time and anthropometry in groups of modern pentathlon adolescents who started training before and after menarche; To analyze the relationships between chronological age and anthropometry in adolescent athletes of modern pentathlon without menarche. 31 athletes with menarche ( $14.69 \pm 2,03$ years old $)$ and 15 without menarche $(12.22$ $\pm 1,07$ years old). Menarche age, gynecological age, anthropometric measurements and the training were assessed. We used descriptive statistics, comparisons for independent samples and Pearson's correlation $(p<0.05)$. The age at menarche was $11.80 \pm 0.95$ years. After division of the group with menarche, a significant difference ( $p=0.006$ ) was observed in age of menarche between girls who started training before $(12.62 \pm 0.95)$ and after the menarche $(11.42 \pm 1.17)$. The descriptive analysis revealed the chronological age of the group whitout menarche being longer than the age of menarche in the groups whit menarche and was greater training time and lower adiposity. The age of onset of training appears to be the greater responsible for the characteristics of menarche presented in pentathletes without discard possible effects of lower body fat percentage.

KEYwords: Sexual Maturity; Anthropometry; Menstrual Cycle; Sports.

\section{Referências}

1. Klug DP, Fonseca PHS. Análise da maturação feminina: um enfoque na idade de ocorrência da menarca. Rev Educ Fís. 2006;17(2):139-147.

2. Al-Sahab B, Ardern CL, Hamadeh MJ, Tamim H. Age at menarche in Canada: results from theNational Longitudinal Survey of Children \& Youth. BMC Public Health. 2010;10(11):1-8.

3. Junqueira ML, Lopes CS, Werneck GL. Family sócio-economic background modified secular trends in age at menarche: evidence from the Pro-Saude Study (Rio de Janeiro, Brazil). Ann Hum Biol. 2003;30(3):347-52.

4. Malina RM, Bouchard C, Bar-Or O. Growth, maturation, and physical activity. Champaign Illinois: Human Kinetics; 2004.

5. Muñoz MT, Piedra C, Barrios V, Garrido G, Argente J. Changes in bone density and bone markers in rhythmic gymnasts and ballet dancers: implications for puberty and leptin levels. Eur J Endocrinol. 2004;151(4):491-496.

6. Kasa-Vubu JZ, Borer KT, Rosenthal A, Meckmongkol T. Twenty-four hour growth hormone and leptin secretion in active postpubertal adolescent girls: impact of fitness, fatness, and age at menarche. J Clin Endocrinol Metab. 2006;91(10):3925-40.

7. Meira TB, Nunomura M. Interação entre a leptina, ginástica artística, puberdade e exercício em atletas do sexo feminino. Rev Bras Ciênc Esporte. 2010;32(1):185-99.

8. Schtscherbyna A, Barreto T, Oliveira FP, Raggio R, Soares EA, Ribeiro BG. A idade do início do treinamento, e não a composição corporal está associada com disfunçôes menstruais em nadadoras adolescents. Rev Bras Med Esporte. 2010;18(3):161-163.

9. Claessens AL, Bourgois J, Beunen G, Philippaert R, Thomis M, Lefevre J, et al. Age at menarche in relation to anthropometric characteristics, competition level and boat category in elite Junior rowers. Ann Hum Biol. 2003;30(2):148-159.

10. Mishra GD, Cooper R, Tom SE, Kuh D. Early life circumstances and their impact on menarche and menopause. Womens Health (Lond Engl). 2009;5(2):175-190.

11. Schtscherbyna A, Soares EA, Oliveira FP, Ribeiro BG. Female athlete triad in elite swimmers of the city of Rio de Janeiro, Brazil. Nutrition. 2009;25(6):634-9.

12. Erlandson MC, Sherar LB, Mirwald RL, Maffulli N, Baxter-Jones AD. Growth and maturation of adolescent female gymnast swimmers and tennis players. Med Sci Sports Exerc. 2008;40(1):34-42.

13. Santos MAM, Leandro CG, Guimarães FJS. Composição corporal e maturação somática de meninas atletas e nãoatletas de natação da cidade do Recife, Brasil. Rev Bras Saúde Matern Infant. 2007;7(2):175-181.

14. Fonseca Junior SJ, Fernandes Filho J. A menarca e seu impacto nas qualidades físicas de escolares. Rev Salud Publica. 2013;15(2):281-293.

15. Jones MA, Hitchen PJ, Stratton G. The importance of considering biological maturity when assessing physical fitness 
measures in girl and boys aged 10 to 16 years. Ann Hum Biol. 2000;27(1):57-65.

16. Malina RM, Ignasiak C, Rozek K, Slawinska T, Domaradzki J, Fugiel J, et al. Growth, maturity and Functional characteristics of female athletes 11-15 years of age. Human Movement. 2011;12(1):31-40.

17. Barkai H, Nichols JF, Rauh MJ, Barrack MT, Lawson MJ, Levy SS. Influence of sports participation and menarche on bone mineral density of female high school athletes. J Sci Med Sport. 2007;10(3):170-179.

18. Lohman T, Roche A, Martorell R. Anthropometric Standartization Reference Manual. Champaign Ilinois: Human Kinetics; 1988.

19. Slaughter MH, Lohman TG, Boileau CA, Horswill CA, Stillman A, Van Loan MD, et al. Skinfold equations for estimation of body fatness in children and youth. Hum Biol. 1988;60(5):709-723.

20. Durnin JVGA, Womersley J. Body fat assessed from total density and its estimation from skinfold thickness: measurements on 481 men and women aged from 16 to 72 years. Br J Nutr. 1974;32(1):77-97.

21. Bacchi E, Spiazzi G, Zendrini G, Bonin C, Moghetti P. Low body weight and menstrual dysfunction are common findings in both elite and amateur ballet dancers. J Endocrinol Invest. 2013;36(5):343-6.

22. Dadgostar H, Razi M, Aleyasin A, Alenabi T, Dahaghin S. The relation between athletic sports and prevalence of amenorrhea and oligomenorrhea in Iranian female athletes. Sports Med, Arthrosc, Rehabil, Ther Technol. 2009;1(1):17.

23. Pardine DP. Alterações hormonais da mulher atleta. Arq Bras Endocrinol Metab. 2001;45(4):343-51.

24. Torstveit MK, Sundgot-Borgen J. Participation in leannes sports but not trainning volume is associated with menstrual dysfunction: a national survey of 1276 elite athletes and controls. Br J Sports Med. 2005;39:141-147.

25. Coelho GM, Gomes AL, Ribeiro BG, Soares EA. Prevention of eating disorders in female athletes. J Sports Med. 2014;12(5):105-13.

26. Meira TB, Moraes FL, Böhme MTS. Relações entre leptina, puberdade e exercício no sexo feminino. Rev Bras Med Esporte. 2009;15(4):306-310.

27. Lassek WD, Gaulin SJ. Menarche is related to fat distribuition. Am J Phys Anthropol. 2007;133(4):1147-1151.

28. Rowland TW. Children's exercise physiology. Champaign Illinois: Human Kinetics; 2005.

ENDEREÇO DE CORRESPONDÊNCIA: Sidnei Jorge Fonseca Junior Estrada do Barro Vermelho, 1695, blE, ap304 - Irajá Rio de Janeiro - RJ - BRASIL CEP: 21540-501 E-mail: sjfjunior@gmail.com.
Submetido: 22/07/2015

Revisado: $12 / 11 / 2017$

Aceito: $20 / 12 / 2017$ 